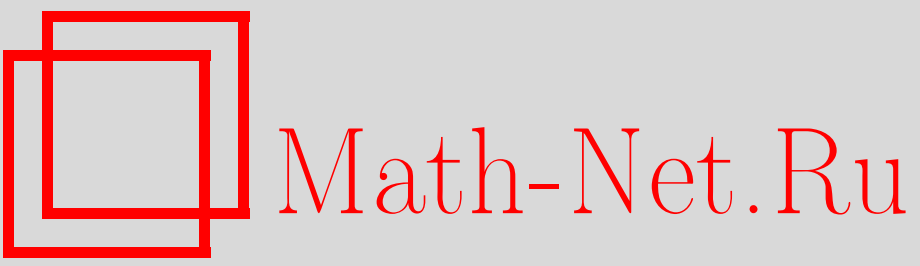

S. Bahlali, Necessary and sufficient conditions of optimality for optimal control problems of forward and backward systems, Теория вероятн. и ее примен., 2009, том 54, выпуск 4, 730-749

DOI: https://doi.org/10.4213/tvp3537

Использование Общероссийского математического портала Math-Net.Ru подразумевает, что вы прочитали и согласны с пользовательским соглашением

http://www . mathnet.ru/rus/agreement

Параметры загрузки:

IP: 54.198 .55 .26

26 апреля 2023 г., 13:42:17 
2009

(C) 2009 г.

BAHLALI S.*

\title{
NECESSARY AND SUFFICIENT CONDITIONS OF OPTIMALITY FOR OPTIMAL CONTROL PROBLEMS OF FORWARD AND BACKWARD SYSTEMS ${ }^{1)}$
}

\begin{abstract}
Мы рассматриваем задачу стохастического управления, где множество значений управления выпукло и система управляется нелинейным прямым и обратным стохастическим дифференциальным уравнением. Мы выводим необходимые и достаточные условия оптимальности в виде стохастического принципа максимума. Результаты сформулированы в слабой форме. При дополнительных предположениях мы получаем эти результаты в глобальной форме. Мы применяем нашу версию стохастического принципа максимума к финансовой модели задачи оценки потока наличных (cash flow valuation problem).

Ключевые слова и фразы: прямое и обратное стохастическое дифференциальное уравнение, стохастический принцип максимума, оптимальное управление, сопряженное уравнение, вариационное уравнение.
\end{abstract}

1. Introduction. We consider a stochastic control problem, where the control domain is convex and the system is governed by a forward and backward stochastic differential equation (FBSDE for short) of the type

$$
\begin{aligned}
d x_{t} & =b\left(t, x_{t}, v_{t}\right) d t+\sigma\left(t, x_{t}, v_{t}\right) d W_{t}, & & x_{0}=x, \\
d y_{t} & =-f\left(t, x_{t}, y_{t}, z_{t}, v_{t}\right) d t+z_{t} d W_{t}, & & y_{T}=\varphi\left(x_{T}\right),
\end{aligned}
$$

where $b, \sigma, f$, and $\varphi$ are given functions, $W=\left(W_{t}\right)_{t \geqslant 0}$ is a standard Brownian motion, defined on a filtered probability space $\left(\Omega, \mathscr{F},\left(\mathscr{F}_{t}\right)_{t \geqslant 0}, \mathbf{P}\right)$ satisfying the usual conditions. The control variable $v=\left(v_{t}\right)$ is a progressively measurable process with values in a convex closed subset of $\mathbf{R}^{k}$.

The objective of the control problem is to choose $v$ in such a way as to minimize a functional, with initial and terminal values, of the type

$$
J(v)=\mathbf{E}\left[g\left(x_{T}\right)+h\left(y_{0}\right)+\int_{0}^{T} l\left(t, x_{t}, y_{t}, z_{t}, v_{t}\right) d t\right]
$$

\footnotetext{
${ }^{*}$ Laboratory of Applied Mathematics, University Med Khider, P.O. Box 145, Biskra 07000, Algeria; e-mail: sbahlali@yahoo.fr

1) This work is supported by Algerian-French cooperation (Tassili 07 MDU 705), and Marie Curie Training Network (ITN) Project.
} 
where $g, h$, and $l$ are given maps.

A control process that solves this problem is called optimal.

The maximum principle for control problems of stochastic differential equations have been studied by many authors including Arkin and Saksonov [3], Bensoussan [9], Haussmann [18], [19], Kushner [23] and Peng [30]. We would like to note that the first papers on the subject have been published by Kushner [23] for the case of uncontrolled diffusion and Arkin and Saksonov [3] in the general case, where the control variable enters both the drift and diffusion coefficients. These works are regarded as the references on the control of the stochastic differential equations.

On the other hand, stochastic control problems for the backward and forward-backward systems have been studied by many authors including Peng [30], Xu [33], El Karoui, Peng, and Quenez [16], Wu [32], Dokuchaev and Zhou [12], Peng and $\mathrm{Wu}$ [31] and Bahlali and Labed [7]. The dynamic programming approach to this kind of problem was developed by Fuhrman and Tessitore [17].

The common fact in the previous results on the control of forwardbackward systems is that the authors consider a constant terminal condition of the backward equation (see [31] and [32]), or a restricted cost (see [33]).

Our aim in this paper is to derive necessary and sufficient conditions of optimality, in the form of the Pontryagin maximum principle. These results are given when the terminal condition of the backward equation depends on the terminal state of the forward equation and the criterion to be minimized is in the general form, with initial and terminal costs.

The main idea is to introduce three adjoint processes to establish necessary and sufficient conditions of optimality. This is a construction of a new method for this kind of problem.

Since the control domain is convex, the classical way to achieve our goal is to use the convex perturbation method. More precisely, if $u$ is an optimal control and $v$ is arbitrary, then we define a perturbed control as follows:

$$
u^{\theta}=u+\theta(v-u) .
$$

We derive the variational equation from the state equation, and the variational inequality from the fact that

$$
0 \leqslant J\left(u^{\theta}\right)-J(u) .
$$

The optimality conditions are then given in weak form. Moreover, under additional hypotheses, we give the results in the form of global stochastic maximum principle.

The special case, where the terminal condition $y_{T}=\xi$ is a measurable random vector, is studied and we prove that in this case stochastic maximum principle is derived with only two adjoint processes. 
Finally, we give an application in financial market. We study the cash flow valuation problem and we prove that the system for this example is naturally governed by a controlled forward and backward stochastic differential equation.

The paper is organized as follows. In Section 2, we formulate the problem and give the various assumptions used throughout the paper. Section 3 is devoted to some preliminary results, which will be used in the sequel. In Section 4, we introduce the adjoint equations and we derive the necessary conditions of optimality. Section 5 treats with the sufficient conditions of optimality. In Section 6, we reformulate necessary and sufficient conditions of optimality in the form of a global maximum principle. Section 7 deals with the special case, where the terminal condition is constant. Finally, in the last section, we apply our version of the stochastic maximum principle to the financial model of cash flow valuation problem.

2. Formulation of the problem. Let $\left(\Omega, \mathscr{F},\left(\mathscr{F}_{t}\right)_{t \geqslant 0}, \mathbf{P}\right)$ be a probability space equipped with a filtration satisfying the usual conditions, on which a $d$-dimensional Brownian motion $W=\left(W_{t}\right)_{t \geqslant 0}$ is defined. We assume that $\left(\mathscr{F}_{t}\right)_{t \geqslant 0}$ is the $\mathbf{P}$-augmentation of the natural filtration of $W$.

Let $T$ be a fixed strictly positive real number and $U$ a closed convex subset of $\mathbf{R}^{k}$.

$\mathrm{D}$ e f i n i t i o n 1. An admissible control $v$ is a progressively measurable process with respect to the filtration $\left(\mathscr{F}_{t}\right)_{t \geqslant 0}$ and with values in $U$ such that

$$
\sup _{t \in[0, T]} \mathbf{E}\left|v_{t}\right|^{2}<\infty
$$

We denote by $\mathscr{U}$ the set of all admissible controls.

For any $v \in \mathscr{U}$, we consider the following FBSDE:

$$
\begin{aligned}
d x_{t} & =b\left(t, x_{t}, v_{t}\right) d t+\sigma\left(t, x_{t}, v_{t}\right) d W_{t}, & & x_{0}=x, \\
d y_{t} & =-f\left(t, x_{t}, y_{t}, z_{t}, v_{t}\right) d t+z_{t} d W_{t}, & & y_{T}=\varphi\left(x_{T}\right),
\end{aligned}
$$

where

$$
\begin{aligned}
& b:[0, T] \times \mathbf{R}^{n} \times U \rightarrow \mathbf{R}^{n}, \quad \sigma:[0, T] \times R^{n} \times U \rightarrow \mathscr{M}_{n \times d}(\mathbf{R}), \\
& f:[0, T] \times \mathbf{R}^{n} \times \mathbf{R}^{m} \times \mathscr{M}_{m \times d}(\mathbf{R}) \times U \rightarrow \mathbf{R}^{m}, \quad \varphi: \mathbf{R}^{n} \rightarrow \mathbf{R}^{m} .
\end{aligned}
$$

We define the criterion to be minimized, with initial and final costs, as follows:

$$
J(v)=\mathbf{E}\left[g\left(x_{T}\right)+h\left(y_{0}\right)+\int_{0}^{T} l\left(t, x_{t}, y_{t}, z_{t}, v_{t}\right) d t\right]
$$

where

$$
\begin{aligned}
& g: \mathbf{R}^{n} \rightarrow \mathbf{R}, \quad h: \mathbf{R}^{m} \rightarrow \mathbf{R}, \\
& l:[0, T] \times \mathbf{R}^{n} \times \mathbf{R}^{m} \times \mathscr{M}_{m \times d}(\mathbf{R}) \times U \rightarrow \mathbf{R} .
\end{aligned}
$$


The control problem is to minimize the functional $J$ over $\mathscr{U}$. A control $u \in \mathscr{U}$ is called optimal, if it solves

$$
J(u)=\inf _{v \in \mathscr{U}} J(v)
$$

Our goal in this paper is to establish necessary and sufficient conditions of optimality in the form of stochastic maximum principle.

We assume that

$b, \sigma, f, g, h, l, \varphi$ are continuously differentiable w.r.t. $(x, y, z, v)$, they are bounded by $C(1+|x|+|y|+|z|+|v|)$, and

their derivatives w.r.t. $(x, y, z, v)$ are continuous and bounded.

Under the above assumptions, for every $v \in \mathscr{U}$, equation (1) has a unique solution and the functional $J$ is well defined from $\mathscr{U}$ into $\mathbf{R}$.

3. Preliminary results. Since the control domain $\mathscr{U}$ is convex, a classical way of treating such a problem is to use the convex perturbation method. More precisely, let $u$ be an optimal control and $\left(x_{t}, y_{t}, z_{t}\right)$ the associated optimal trajectory and define a perturbed control as follows:

$$
u_{t}^{\theta}=u_{t}+\theta\left(v_{t}-u_{t}\right),
$$

where $\theta>0$ is sufficiently small and $v$ is an arbitrary element of $\mathscr{U}$.

The perturbed control $u^{\theta}$ is admissible and denote by $\left(x_{t}^{\theta}, y_{t}^{\theta}, z_{t}^{\theta}\right)$ the trajectory of the system associated with $u^{\theta}$.

From optimality of $u$, the variational inequality of the Hamiltonian will be derived from the fact that

$$
0 \leqslant J\left(u^{\theta}\right)-J(u)
$$

For this end, we need the following classical lemmas.

Lemma 1. Under assumptions (4), we have

$$
\begin{gathered}
\lim _{\theta \rightarrow 0}\left[\sup _{0 \leqslant t \leqslant T} \mathbf{E}\left|x_{t}^{\theta}-x_{t}\right|^{2}\right]=0, \\
\lim _{\theta \rightarrow 0}\left[\sup _{0 \leqslant t \leqslant T} \mathbf{E}\left|y_{t}^{\theta}-y_{t}\right|^{2}\right]=0, \\
\lim _{\theta \rightarrow 0} \mathbf{E} \int_{0}^{T}\left|z_{t}^{\theta}-z_{t}\right|^{2} d t=0 .
\end{gathered}
$$

$\mathrm{P}$ r o o f. Since $b$ and $\sigma$ are Lipschitz with respect to $(x, v)$, we have

$$
\mathbf{E}\left|x_{t}^{\theta}-x_{t}\right|^{2} \leqslant C \int_{0}^{t} \mathbf{E}\left|x_{s}^{\theta}-x_{s}\right|^{2} d s+C \theta^{2} \int_{0}^{t} \mathbf{E}\left|v_{s}-u_{s}\right|^{2} d s .
$$


By using the Gronwall lemma and Definition 1, we get

$$
\lim _{\theta \rightarrow 0} \mathbf{E}\left|x_{t}^{\theta}-x_{t}\right|^{2}=0 .
$$

Applying the Burkholder-Davis-Gundy inequality for the martingale part allows us to obtain a uniform convergence in $t$ :

$$
\lim _{\theta \rightarrow 0}\left[\sup _{0 \leqslant t \leqslant T} \mathbf{E}\left|x_{t}^{\theta}-x_{t}\right|^{2}\right]=0 .
$$

This prove (7).

Let us now prove (8) and (9).

By applying the Itô formula to $\left(y_{t}^{\theta}-y_{t}\right)^{2}$, we have

$$
\begin{aligned}
& \mathbf{E}\left|y_{t}^{\theta}-y_{t}\right|^{2}+\mathbf{E} \int_{t}^{T}\left|z_{t}^{\theta}-z_{t}\right|^{2} d s=\mathbf{E}\left|\varphi\left(x_{T}^{\theta}\right)-\varphi\left(x_{T}\right)\right|^{2} \\
& \quad+2 \mathbf{E} \int_{t}^{T}\left|\left(y_{s}^{\theta}-y_{s}\right)\left[f\left(s, x_{s}^{\theta}, y_{s}^{\theta}, z_{s}^{\theta}, u_{s}^{\theta}\right)-f\left(s, x_{s}, y_{s}, z_{s}, u_{s}\right)\right]\right| d s .
\end{aligned}
$$

By the Young formula, for every $\varepsilon>0$, we have

$$
\begin{aligned}
\mathbf{E} & \left|\left(y_{s}^{\theta}-y_{s}\right)\left[f\left(s, x_{s}^{\theta}, y_{s}^{\theta}, z_{s}^{\theta}, u_{s}^{\theta}\right)-f\left(s, x_{s}, y_{s}, z_{s}, u_{s}\right)\right]\right| \\
\leqslant & \frac{1}{2 \varepsilon} \mathbf{E}\left|y_{s}^{\theta}-y_{s}\right|^{2}+\frac{\varepsilon}{2} \mathbf{E}\left|f\left(s, x_{s}^{\theta}, y_{s}^{\theta}, z_{s}^{\theta}, u_{s}^{\theta}\right)-f\left(s, x_{s}, y_{s}, z_{s}, u_{s}\right)\right|^{2}, \\
\leqslant & \left(\frac{1}{2 \varepsilon}+\frac{C \varepsilon}{2}\right) \mathbf{E}\left|y_{s}^{\theta}-y_{s}\right|^{2}+\frac{C \varepsilon}{2} \mathbf{E}\left|z_{s}^{\theta}-z_{s}\right|^{2} \\
& \quad+\frac{C \varepsilon}{2} \mathbf{E}\left|x_{s}^{\theta}-x_{s}\right|^{2}+\frac{C \varepsilon \theta}{2} \mathbf{E}\left|v_{s}-u_{s}\right|^{2} .
\end{aligned}
$$

Thus, (10) becomes

$$
\begin{aligned}
\mathbf{E}\left|y_{t}^{\theta}-y_{t}\right|^{2}+\mathbf{E} \int_{t}^{T}\left|z_{s}^{\theta}-z_{s}\right|^{2} d s \leqslant & \left(C \varepsilon+\frac{1}{\varepsilon}\right) \int_{t}^{T} \mathbf{E}\left|y_{s}^{\theta}-y_{s}\right|^{2} d s \\
& +C \varepsilon \int_{t}^{T} \mathbf{E}\left|z_{s}^{\theta}-z_{s}\right|^{2} d s+\alpha_{t}^{\theta}
\end{aligned}
$$

where $\alpha_{t}^{\theta}=\mathbf{E}\left|x_{T}^{\theta}-x_{T}\right|^{2}+C \varepsilon\left(\mathbf{E} \int_{t}^{T}\left|x_{s}^{\theta}-x_{s}\right|^{2} d s+\theta^{2} \mathbf{E} \int_{t}^{T}\left|v_{s}-u_{s}\right|^{2} d s\right)$.

By Definition 1 and (7), we get

$$
\lim _{\theta \rightarrow 0} \alpha_{t}^{\theta}=0 .
$$

Choose $\varepsilon=1 /(2 C)$. Then (11) becomes

$$
\mathbf{E}\left|y_{t}^{\theta}-y_{t}\right|^{2}+\frac{1}{2} \mathbf{E} \int_{t}^{T}\left|z_{s}^{\theta}-z_{s}\right|^{2} d s \leqslant\left(2 C+\frac{1}{2}\right) \int_{t}^{T} \mathbf{E}\left|y_{s}^{\theta}-y_{s}\right|^{2} d s+\alpha_{t}^{\theta} .
$$


From the above inequality, we derive two inequalities

$$
\begin{aligned}
\mathbf{E}\left|y_{t}^{\theta}-y_{t}\right|^{2} & \leqslant\left(2 C+\frac{1}{2}\right) \int_{t}^{T} \mathbf{E}\left|y_{s}^{\theta}-y_{s}\right|^{2} d s+\alpha_{t}^{\theta}, \\
\mathbf{E} \int_{t}^{T}\left|z_{s}^{\theta}-z_{s}\right|^{2} d s & \leqslant(4 C+1) \int_{t}^{T} \mathbf{E}\left|y_{s}^{\theta}-y_{s}\right|^{2} d s+2 \alpha_{t}^{\theta} .
\end{aligned}
$$

By using (7), (13), the Gronwall lemma, and the Burkholder-DavisGundy inequality, we get

$$
\lim _{\theta \rightarrow 0} \mathbf{E}\left|y_{t}^{\theta}-y_{t}\right|^{2}=0
$$

To get the result with sup norm (i.e., (8)), it is sufficient to apply the Burkholder-Davis-Gundy inequality to the martingale part in the Itô formula. We have then (8). Finally, from (8) and (12) we obtain (9). Lemma 1 is proved.

Lemma 2. Let $\widetilde{x}_{t}$ and $\widetilde{y}_{t}$ be respectively the solutions of the following linear equations:

$$
\begin{aligned}
d \widetilde{x}_{t}= & {\left[b_{x}\left(t, x_{t}, u_{t}\right) \widetilde{x}_{t}+b_{v}\left(t, x_{t}, u_{t}\right)\left(v_{t}-u_{t}\right)\right] d t } \\
& +\left[\sigma_{x}\left(t, x_{t}, u_{t}\right) \widetilde{x}_{t}+\sigma_{v}\left(t, x_{t}, u_{t}\right)\left(v_{t}-u_{t}\right)\right] d W_{t}, \\
\widetilde{x}_{0}= & 0
\end{aligned}
$$

$$
\begin{aligned}
d \widetilde{y}_{t}= & -\left[f_{x}\left(t, x_{t}, y_{t}, z_{t}, u_{t}\right) \widetilde{x}_{t}+f_{y}\left(t, x_{t}, y_{t}, z_{t}, u_{t}\right) \widetilde{y}_{t}\right] d t \\
& -\left[f_{z}\left(t, x_{t}, y_{t}, z_{t}, u_{t}\right) \widetilde{z}_{t}+f_{v}\left(t, x_{t}, y_{t}, z_{t}, u_{t}\right)\left(v_{t}-u_{t}\right)\right] d t+\widetilde{z}_{t} d W_{t}, \\
\widetilde{y}_{T}= & \varphi_{x}\left(x_{T}\right) \widetilde{x}_{T} .
\end{aligned}
$$

Then

$$
\begin{aligned}
\lim _{\theta \rightarrow 0} \mathbf{E}\left|\widetilde{x}_{t}-\frac{x_{t}^{\theta}-x_{t}}{\theta}\right|^{2} & =0 \\
\lim _{\theta \rightarrow 0} \mathbf{E}\left|\widetilde{y}_{t}-\frac{y_{t}^{\theta}-y_{t}}{\theta}\right|^{2} & =0 \\
\lim _{\theta \rightarrow 0} \mathbf{E} \int_{0}^{T}\left|\widetilde{z}_{t}-\frac{z_{t}^{\theta}-z_{t}}{\theta}\right|^{2} d t & =0 .
\end{aligned}
$$

P r o o f. For simplicity, we put

$$
X_{t}=\widetilde{x}_{t}-\frac{x_{t}^{\theta}-x_{t}}{\theta}, \quad Y_{t}=\widetilde{y}_{t}-\frac{y_{t}^{\theta}-y_{t}}{\theta}, \quad Z_{t}=\widetilde{z}_{t}-\frac{z_{t}^{\theta}-z_{t}}{\theta},
$$

and

$$
\Lambda_{t}^{\theta}=\left(t, x_{t}+\lambda \theta\left(\widetilde{x}_{t}-X_{t}\right), y_{t}+\lambda \theta\left(\widetilde{y}_{t}-Y_{t}\right), z_{t}+\lambda \theta\left(\widetilde{z}_{t}-Z_{t}\right), u_{t}+\lambda \theta\left(v_{t}-u_{t}\right)\right) .
$$


We have

$$
\begin{aligned}
X_{t}= & \int_{0}^{t} \int_{0}^{1} b_{x}\left(s, x_{s}+\lambda \theta\left(\widetilde{x}_{s}-X_{s}\right), u_{s}+\lambda \theta\left(v_{s}-u_{s}\right)\right) X_{s} d \lambda d s \\
& +\int_{0}^{t} \int_{0}^{1} \sigma_{x}\left(s, x_{s}+\lambda \theta\left(\widetilde{x}_{s}-X_{s}\right), u_{s}+\lambda \theta\left(v_{s}-u_{s}\right)\right) X_{s} d \lambda d W_{s}+\beta_{t}^{\theta}
\end{aligned}
$$

where

$$
\begin{aligned}
\beta_{t}^{\theta}= & \int_{0}^{t} b_{x}\left(s, x_{s}, u_{s}\right) \widetilde{x}_{s} d s+\int_{0}^{t} \sigma_{x}\left(s, x_{s}, u_{s}\right) \widetilde{x}_{s} d W_{s} \\
& +\int_{0}^{t} b_{v}\left(s, x_{s}, u_{s}\right)\left(v_{s}-u_{s}\right) d s+\int_{0}^{t} \sigma_{v}\left(s, x_{s}, u_{s}\right)\left(v_{s}-u_{s}\right) d W_{s} \\
& -\int_{0}^{t} \int_{0}^{1} b_{x}\left(s, x_{s}+\lambda \theta\left(\widetilde{x}_{s}-X_{s}\right), u_{s}+\lambda \theta\left(v_{s}-u_{s}\right)\right) \widetilde{x}_{s} d \lambda d s \\
& -\int_{0}^{t} \int_{0}^{1} \sigma_{x}\left(s, x_{s}+\lambda \theta\left(\widetilde{x}_{s}-X_{s}\right), u_{s}+\lambda \theta\left(v_{s}-u_{s}\right)\right) \widetilde{x}_{s} d \lambda d W_{s} \\
& -\int_{0}^{t} \int_{0}^{1} b_{v}\left(s, x_{s}+\lambda \theta\left(\widetilde{x}_{s}-X_{s}\right), u_{s}+\lambda \theta\left(v_{s}-u_{s}\right)\right)\left(v_{s}-u_{s}\right) d \lambda d s \\
& -\int_{0}^{t} \int_{0}^{1} \sigma_{v}\left(s, x_{s}+\lambda \theta\left(\widetilde{x}_{s}-X_{s}\right), u_{s}+\lambda \theta\left(v_{s}-u_{s}\right)\right)\left(v_{s}-u_{s}\right) d \lambda d W_{s} .
\end{aligned}
$$

Since the derivatives $b_{x}$ and $\sigma_{x}$ are bounded, we have $\mathbf{E}\left|X_{t}\right|^{2}=$ $C \mathbf{E} \int_{0}^{t}\left|X_{s}\right|^{2} d s+C \mathbf{E}\left|\beta_{t}^{\theta}\right|^{2}$. Since $b_{x}, \sigma_{x}, b_{v}, \sigma_{v}$ are continuous and bounded, we have $\lim _{\theta \rightarrow 0} \beta_{t}^{\theta}=0$.

By using the Gronwall lemma, we obtain (17).

Let us now prove (18) and (19).

We have

$$
d Y_{t}=-\left(F_{t}^{y} Y_{t}+F_{t}^{z} Z_{t}-\gamma_{t}^{\theta}\right) d t+Z_{t} d W_{t}
$$

where

$$
F_{t}^{y}=\int_{0}^{1} f_{y}\left(\Lambda_{t}^{\theta}\right) d \lambda, \quad F_{t}^{z}=\int_{0}^{1} f_{z}\left(\Lambda_{t}^{\theta}\right) d \lambda
$$

and $\gamma_{t}^{\theta}$ is given by

$$
\begin{aligned}
\gamma_{t}^{\theta}= & \int_{0}^{1} f_{x}\left(\Lambda_{t}^{\theta}\right) \widetilde{x} d \lambda+\int_{0}^{1} f_{y}\left(\Lambda_{t}^{\theta}\right) \widetilde{y}_{t} d \lambda+\int_{0}^{1} f_{z}\left(\Lambda_{t}^{\theta}\right) \widetilde{z} d \lambda \\
& +\int_{0}^{1} f_{v}\left(\Lambda_{t}^{\theta}\right)\left(v_{t}-u_{t}\right) d \lambda-\int_{0}^{1} f_{x}\left(\Lambda_{t}^{\theta}\right) X_{t} d \lambda \\
& -f_{x}\left(t, x_{t}, y_{t}, z_{t}, u_{t}\right) \widetilde{x}_{t}-f_{y}\left(t, x_{t}, y_{t}, z_{t}, u_{t}\right) \widetilde{y}_{t} \\
& -f_{z}\left(t, x_{t}, y_{t}, z_{t}, u_{t}\right) \widetilde{z}_{t}-f_{v}\left(t, x_{t}, y_{t}, z_{t}, u_{t}\right)\left(v_{t}-u_{t}\right) .
\end{aligned}
$$

By applying the Itô formula to $\left(Y_{t}\right)^{2}$, we find that

$$
\mathbf{E}\left|Y_{t}\right|^{2}+\mathbf{E} \int_{t}^{T}\left|Z_{s}\right|^{2} d s=\mathbf{E}\left|Y_{T}\right|^{2}+2 \mathbf{E} \int_{t}^{T}\left|Y_{s}\left(F_{s}^{y} Y_{s}+F_{s}^{z} Z_{s}-\gamma_{s}^{\theta}\right)\right| d s .
$$


By the Young formula, for every $\varepsilon>0$, we obtain

$$
\begin{aligned}
\mathbf{E}\left|Y_{s}\left(F_{s}^{y} Y_{s}+F_{s}^{z} Z_{s}-\gamma_{s}^{\theta}\right)\right| \leqslant & \frac{1}{2 \varepsilon} \mathbf{E}\left|Y_{s}\right|^{2}+\frac{\varepsilon}{2} \mathbf{E}\left|F_{s}^{y} Y_{s}+F_{s}^{z} Z_{s}-\gamma_{s}^{\theta}\right|^{2} \\
\leqslant & \frac{1}{2 \varepsilon} \mathbf{E}\left|Y_{s}\right|^{2}+\frac{C \varepsilon}{2} \mathbf{E}\left|F_{s}^{y} Y_{s}\right|^{2} \\
& +\frac{C \varepsilon}{2} \mathbf{E}\left|F_{s}^{z} Z_{s}\right|^{2}+\frac{C \varepsilon}{2} \mathbf{E}\left|\gamma_{s}^{\theta}\right|^{2} .
\end{aligned}
$$

Since $F_{t}^{y}$ and $F_{t}^{z}$ are bounded, we have

$\mathbf{E}\left|Y_{s}\left(F_{s}^{y} Y_{s}+F_{s}^{z} Z_{s}-\gamma_{s}^{\theta}\right)\right| \leqslant\left(\frac{1}{2 \varepsilon}+\frac{C \varepsilon}{2}\right) \mathbf{E}\left|Y_{s}\right|^{2}+\frac{C \varepsilon}{2} \mathbf{E}\left|Z_{s}\right|^{2}+\frac{C \varepsilon}{2} \mathbf{E}\left|\gamma_{s}^{\theta}\right|^{2}$.

Thus, we can rewrite (21) as follows:

$$
\begin{aligned}
\mathbf{E}\left|Y_{t}\right|^{2}+\mathbf{E} \int_{t}^{T}\left|Z_{s}\right|^{2} d s \leqslant & \left(C \varepsilon+\frac{1}{\varepsilon}\right) \int_{t}^{T} \mathbf{E}\left|Y_{s}\right|^{2} d s \\
& +C \varepsilon \int_{t}^{T} \mathbf{E}\left|Z_{s}\right|^{2} d s+\delta_{t}^{\theta}
\end{aligned}
$$

where

$$
\delta_{t}^{\theta}=\mathbf{E}\left|Y_{T}\right|^{2}+C \varepsilon \mathbf{E} \int_{t}^{T}\left|\gamma_{s}^{\theta}\right|^{2} d s .
$$

Choose $\varepsilon=1 /(2 C)$, then (22) becomes

$$
\mathbf{E}\left|Y_{t}\right|^{2}+\frac{1}{2} \mathbf{E} \int_{t}^{T}\left|Z_{s}\right|^{2} d s \leqslant\left(2 C+\frac{1}{2}\right) \int_{t}^{T} \mathbf{E}\left|Y_{s}\right|^{2} d s+\delta_{t}^{\theta} .
$$

From the above inequality, we deduce two inequalities

$$
\begin{array}{r}
\mathbf{E}\left|Y_{t}\right|^{2} \leqslant\left(2 C+\frac{1}{2}\right) \int_{t}^{T} \mathbf{E}\left|Y_{s}\right|^{2} d s+\delta_{t}^{\theta}, \\
\mathbf{E} \int_{t}^{T}\left|Z_{s}\right|^{2} d s \leqslant(4 C+1) \int_{t}^{T} \mathbf{E}\left|Y_{s}\right|^{2} d s+2 \delta_{t}^{\theta} .
\end{array}
$$

From (20) and the continuity and boundedness of $f_{x}, f_{y}, f_{z}, f_{v}$, we deduce that

$$
\lim _{\theta \rightarrow 0} \gamma_{t}^{\theta}=0
$$

On the other hand, we have

$$
\begin{aligned}
\mathbf{E}\left|Y_{T}\right|^{2}= & \mathbf{E}\left|\widetilde{y}_{T}-\frac{y_{T}^{\theta}-y_{T}}{\theta}\right|^{2}=\mathbf{E}\left|\varphi_{x}\left(x_{T}\right) \widetilde{x}_{T}-\frac{\varphi\left(x_{T}^{\theta}\right)-\varphi\left(x_{T}\right)}{\theta}\right|^{2} \\
\leqslant & 2 \mathbf{E}\left|\varphi_{x}\left(x_{T}\right) \widetilde{x}_{T}-\int_{0}^{1} \varphi_{x}\left(x_{T}+\lambda \theta\left(\widetilde{x}_{T}-X_{T}\right)\right) \widetilde{x}_{T} d \lambda\right|^{2} \\
& +2 \mathbf{E} \int_{0}^{1}\left|\varphi_{x}\left(x_{T}+\lambda \theta\left(\widetilde{x}_{T}-X_{T}\right)\right) X_{T}\right|^{2} d \lambda .
\end{aligned}
$$


Since $\varphi_{x}$ is continuous and bounded, we obtain from (17) that

$$
\lim _{\theta \rightarrow 0} \mathbf{E}\left|Y_{T}\right|^{2}=0 .
$$

By (23), (26), and (27), we deduce

$$
\lim _{\theta \rightarrow 0} \delta_{t}^{\theta}=0
$$

By using (24), (28), the Gronwall lemma, and the Burkholder-DavisGundy inequality, we obtain (18). Finally, (19) is derived from (18), (25), and (28). Lemma 2 is proved.

Lemma 3. Let $u$ be an optimal control minimizing the functional $J$ over $\mathscr{U}$ and $\left(x_{t}, y_{t}, z_{t}\right)$ its associated trajectory. Then for any $v \in \mathscr{U}$ we have

$$
\begin{gathered}
0 \leqslant \mathbf{E}\left[g_{x}\left(x_{T}\right) \widetilde{x}_{T}\right]+\mathbf{E}\left[h_{y}\left(y_{0}\right) \widetilde{y}_{0}\right]+\mathbf{E} \int_{0}^{T} l_{v}\left(t, x_{t}, y_{t}, z_{t}, u_{t}\right)\left(v_{t}-u_{t}\right) d t \\
+\mathbf{E} \int_{0}^{T}\left[l_{x}\left(t, x_{t}, y_{t}, z_{t}, u_{t}\right) \widetilde{x}_{t}+l_{y}\left(t, x_{t}, y_{t}, z_{t}, u_{t}\right) \widetilde{y}_{t}\right. \\
\left.\quad+l_{z}\left(t, x_{t}, y_{t}, z_{t}, u_{t}\right) \widetilde{z}_{t}\right] d t .
\end{gathered}
$$

P r o o f. We use the same notation as in the proof of Lemma 2.

From inequality (6), we have

$$
\begin{aligned}
0 \leqslant & \mathbf{E} \int_{0}^{1} g_{x}\left(t, x_{t}+\lambda \theta\left(\widetilde{x}_{t}-X_{t}\right)\right) \widetilde{x}_{t} d \lambda+\mathbf{E} \int_{0}^{1} h_{y}\left(y_{t}+\lambda \theta\left(y_{t}-\widetilde{y}_{t}\right)\right) \widetilde{y}_{t} d \lambda \\
& +\mathbf{E} \int_{0}^{T} \int_{0}^{1}\left[l_{x}\left(\Lambda_{t}^{\theta}\right) \widetilde{x}_{t}+l_{y}\left(\Lambda_{t}^{\theta}\right) \widetilde{y}_{t}+l_{z}\left(\Lambda_{t}^{\theta}\right) \widetilde{z}_{t}+l_{v}\left(\Lambda_{t}^{\theta}\right)\left(v_{t}-u_{t}\right)\right] d \lambda d t-\rho_{t}^{\theta}
\end{aligned}
$$

where

$$
\begin{aligned}
\rho_{t}^{\theta}= & \mathbf{E} \int_{0}^{1} g_{x}\left(t, x_{t}+\lambda \theta\left(\widetilde{x}_{t}-X_{t}\right)\right) X_{t} d \lambda+\mathbf{E} \int_{0}^{1} h_{y}\left(y_{t}+\lambda \theta\left(y_{t}-\widetilde{y}_{t}\right)\right) Y_{t} d \lambda \\
& +\mathbf{E} \int_{0}^{T} \int_{0}^{1}\left[l_{x}\left(\Lambda_{t}^{\theta}\right) X_{t}+l_{y}\left(\Lambda_{t}^{\theta}\right) Y_{t}+l_{y}\left(\Lambda_{t}^{\theta}\right) Z_{t}\right] d \lambda d t .
\end{aligned}
$$

Since the derivatives $g_{x}, h_{y}, l_{x}, l_{y}, l_{z}$, and $l_{v}$ are continuous and bounded, we deduce from (17), (18), and (19) that $\lim _{\theta \rightarrow 0} \rho_{t}^{\theta}=0$.

By letting $\theta$ go to 0 in the above inequality, the proof is completed.

4. Adjoint equations and necessary conditions of optimality. Introduce the following three stochastic differential equations, called adjoints equations:

$$
\begin{aligned}
d p_{t}= & -\left[l_{x}\left(t, x_{t}, y_{t}, z_{t}, u_{t}\right)+b_{x}\left(t, x_{t}, u_{t}\right) p_{t}+\sigma_{x}\left(t, x_{t}, u_{t}\right) P_{t}\right] d t \\
& -f_{x}\left(t, x_{t}, y_{t}, z_{t}, u_{t}\right) q_{t} d t+P_{t} d W_{t}, \quad p_{T}=g_{x}\left(x_{T}\right),
\end{aligned}
$$




$$
\begin{aligned}
d q_{t}= & {\left[l_{y}\left(t, x_{t}, y_{t}, z_{t}, u_{t}\right)+f_{y}\left(t, x_{t}, y_{t}, z_{t}, u_{t}\right) q_{t}\right] d t } \\
& +\left[l_{z}\left(t, x_{t}, y_{t}, z_{t}, u_{t}\right)+f_{z}\left(t, x_{t}, y_{t}, z_{t}, u_{t}\right) q_{t}\right] d W_{t}, \quad q_{0}=h_{y}\left(y_{0}\right) .
\end{aligned}
$$

$d k_{t}=-\left[b_{x}\left(t, x_{t}, u_{t}\right) k_{t}+\sigma_{x}\left(t, x_{t}, u_{t}\right) K_{t}\right] d t+K_{t} d W_{t}, \quad k_{T}=-\varphi_{x}\left(x_{T}\right) q_{T}$.

The processes $p, q$, and $k$ (called adjoint processes) are progressively measurable with respect to the filtration $\left(\mathscr{F}_{t}\right)_{t \geqslant 0}$ and we have

$$
\begin{aligned}
(p, P) & \in L_{\mathscr{F}}^{2}\left([0, T] ; \mathbf{R}^{n}\right) \times L_{\mathscr{F}}^{2}\left([0, T] ; \mathbf{R}^{n \times d}\right), \\
q & \in L_{\mathscr{F}}^{2}\left([0, T] ; \mathbf{R}^{m}\right), \\
(k, K) & \in L_{\mathscr{F}}^{2}\left([0, T] ; \mathbf{R}^{n}\right) \times L_{\mathscr{F}}^{2}\left([0, T] ; \mathbf{R}^{n \times d}\right) .
\end{aligned}
$$

Since $p_{T}=g_{x}\left(x_{T}\right)$ and $q_{0}=h_{y}\left(y_{0}\right)$, inequality (29) becomes

$$
\begin{aligned}
& 0 \leqslant \mathbf{E}\left[p_{T} \widetilde{x}_{T}\right]+\mathbf{E}\left[q_{0} \widetilde{y}_{0}\right]+\mathbf{E} \int_{0}^{T} l_{v}\left(t, x_{t}, y_{t}, z_{t}, u_{t}\right)\left(v_{t}-u_{t}\right) d t \\
& +\mathbf{E} \int_{0}^{T}\left[l_{x}\left(t, x_{t}, y_{t}, z_{t}, u_{t}\right) \widetilde{x}_{t}+l_{y}\left(t, x_{t}, y_{t}, z_{t}, u_{t}\right) \widetilde{y}_{t}\right. \\
& \left.+l_{z}\left(t, x_{t}, y_{t}, z_{t}, u_{t}\right) \tilde{z}_{t}\right] d t .
\end{aligned}
$$

By applying the Itô formula to $\left(p_{t} \widetilde{x}_{t}\right),\left(q_{t} \widetilde{y}_{t}\right)$, we have

$$
\begin{aligned}
\mathbf{E}\left[p_{T} \widetilde{x}_{T}\right]= & -\mathbf{E} \int_{0}^{T}\left[l_{x}\left(t, x_{t}, y_{t}, z_{t}, u_{t}\right) \widetilde{x}_{t}+f_{x}\left(t, x_{t}, y_{t}, z_{t}, u_{t}\right) q_{t} \widetilde{x}_{t}\right] d t \\
& +\mathbf{E} \int_{0}^{T}\left[b_{v}\left(t, x_{t}, u_{t}\right) p_{t}+\sigma_{v}\left(t, x_{t}, u_{t}\right) P_{t}\right]\left(v_{t}-u_{t}\right) d t .
\end{aligned}
$$

$\mathbf{E}\left[q_{0} \widetilde{y}_{0}\right]=\mathbf{E}\left[q_{T} \widetilde{y}_{T}\right]-\mathbf{E} \int_{0}^{T}\left[l_{y}\left(t, x_{t}, y_{t}, z_{t}, u_{t}\right) \widetilde{y}_{t}+l_{z}\left(t, x_{t}, y_{t}, z_{t}, u_{t}\right) \widetilde{z}_{t}\right] d t$

$$
\begin{aligned}
+\mathbf{E} \int_{0}^{T}[ & f_{x}\left(t, x_{t}, y_{t}, z_{t}, u_{t}\right) q_{t} \widetilde{x}_{t} \\
& \left.+f_{v}\left(t, x_{t}, y_{t}, z_{t}, u_{t}\right) q_{t}\left(v_{t}-u_{t}\right)\right] d t .
\end{aligned}
$$

Then (33) becomes

$$
\begin{aligned}
0 \leqslant & \mathbf{E}\left[q_{T} \widetilde{y}_{T}\right]+\mathbf{E} \int_{0}^{T}\left[\left(b_{v}\left(t, x_{t}, u_{t}\right) p_{t}+\sigma_{v}\left(t, x_{t}, u_{t}\right) P_{t}\right)\left(v_{t}-u_{t}\right)\right] d t \\
& +\mathbf{E} \int_{0}^{T}\left[f_{x}\left(t, x_{t}, y_{t}, z_{t}, u_{t}\right) \widetilde{x}_{t} q_{t}+f_{v}\left(t, x_{t}, y_{t}, z_{t}, u_{t}\right)\left(v_{t}-u_{t}\right) q_{t}\right] d t \\
& +\mathbf{E} \int_{0}^{T} l_{v}\left(t, x_{t}, y_{t}, z_{t}, u_{t}\right)\left(v_{t}-u_{t}\right) d t .
\end{aligned}
$$

We remark that $q_{T} \widetilde{y}_{T}=-k_{T} \widetilde{x}_{T}$. Then by applying the Itô formula to $\left(k_{t} \widetilde{x}_{t}\right)$, we have

$\mathbf{E}\left[q_{T} \widetilde{y}_{T}\right]=-\mathbf{E}\left[k_{T} \widetilde{x}_{T}\right]=-\mathbf{E} \int_{0}^{T}\left[b_{v}\left(t, x_{t}, u_{t}\right) k_{t}+\sigma_{v}\left(t, x_{t}, u_{t}\right) K_{t}\right]\left(v_{t}-u_{t}\right) d t$. 
Thus (36) becomes

$$
0 \leqslant \mathbf{E} \int_{0}^{T} H_{v}\left(t, x_{t}, y_{t}, z_{t}, u_{t}, p_{t}, q_{t}, k_{t}\right)\left(u_{t}-v_{t}\right) d t \quad \forall v \in \mathscr{U},
$$

where the Hamiltonian $H$ from $[0, T] \times \mathbf{R}^{n} \times \mathbf{R}^{m} \times \mathscr{M}_{m \times d}(\mathbf{R}) \times U \times \mathbf{R}^{n} \times$ $\mathbf{R}^{m} \times \mathbf{R}^{n}$ into $\mathbf{R}$ is defined by

$$
\begin{aligned}
H(t, x, y, z, v, p, q, k)= & -l(t, x, y, z, v)-b(t, x, v)(p-k) \\
& -\sigma(t, x, v)(P-K)-f(t, x, y, z, v) q .
\end{aligned}
$$

We can now state the necessary conditions of optimality.

Theorem 1. Let $u$ be an optimal control minimizing the functional $J$ over $\mathscr{U}$ and $\left(x_{t}, y_{t}, z_{t}\right)$ the corresponding optimal trajectory. Then there are three unique progressively measurable processes $p, q$, and $k$, which are, respectively, solutions of stochastic differential equations (30), (31), and (32) such that

$$
0 \leqslant H_{v}\left(t, x_{t}, y_{t}, z_{t}, u_{t}, p_{t}, q_{t}, k_{t}\right)\left(u_{t}-v_{t}\right) \quad \forall v \in \mathscr{U}, \text { a.s., a.e. }
$$

P r o o f. The result follows immediately from (37).

5. The sufficient conditions of optimality. In this section, we study when the necessary conditions of optimality (38) becomes sufficient.

Theorem 2 (Sufficient conditions of optimality). Assume that $g(\cdot)$ and $h(\cdot)$ are convex and the map $(x, y, z, v) \mapsto H(t, x, y, z, v, p, q, k)$ is concave.

Then $u$ is an optimal control if it satisfies (38).

P r o o f. Let $u$ be an arbitrary admissible control (candidate to be optimal) and $\left(x_{t}^{u}, y_{t}^{u}, z_{t}^{u}\right)$ the trajectory of the system controlled by $u$. For any admissible control $v$, with associated trajectory $\left(x_{t}^{v}, y_{t}^{v}, z_{t}^{v}\right)$, we have

$$
\begin{aligned}
J(v)-J(u)= & \mathbf{E}\left[g\left(x_{T}^{v}\right)-g\left(x_{T}^{u}\right)\right]+\mathbf{E}\left[h\left(y_{0}^{v}\right)-h\left(y_{0}^{u}\right)\right] \\
& +\mathbf{E} \int_{0}^{T}\left[l\left(t, x_{t}^{v}, y_{t}^{v}, z_{t}^{v}, v_{t}\right)-l\left(t, x_{t}^{u}, y_{t}^{u}, z_{t}^{u}, u_{t}\right)\right] d t .
\end{aligned}
$$

Since $g$ and $h$ are convex, we have

$$
\begin{aligned}
g\left(x_{T}^{v}\right)-g\left(x_{T}^{u}\right) & \geqslant g_{x}\left(x_{T}^{u}\right)\left(x_{T}^{v}-x_{T}^{u}\right), \\
h\left(y_{0}^{v}\right)-h\left(y_{0}^{u}\right) & \geqslant h_{y}\left(y_{0}^{u}\right)\left(y_{0}^{v}-y_{0}^{u}\right) .
\end{aligned}
$$

Then

$$
\begin{aligned}
J(v)-J(u) \geqslant & \mathbf{E}\left[g_{x}\left(x_{T}^{u}\right)\left(x_{T}^{v}-x_{T}^{u}\right)\right]+\mathbf{E}\left[h_{y}\left(y_{0}^{u}\right)\left(y_{0}^{v}-y_{0}^{u}\right)\right] \\
& +\mathbf{E} \int_{0}^{T}\left[l\left(t, x_{t}^{v}, y_{t}^{v}, z_{t}^{v}, v_{t}\right)-l\left(t, x_{t}^{u}, y_{t}^{u}, z_{t}^{u}, u_{t}\right)\right] d t .
\end{aligned}
$$


We remark that $p_{T}=g_{x}\left(x_{T}^{u}\right)$ and $q_{0}=h_{y}\left(y_{0}^{u}\right)$, then

$$
\begin{aligned}
J(v)-J(u) \geqslant & \mathbf{E}\left[p_{T}\left(x_{T}^{v}-x_{T}^{u}\right)\right]+\mathbf{E}\left[q_{0}\left(x_{0}^{v}-y_{0}^{u}\right)\right] \\
& +\mathbf{E} \int_{0}^{T}\left[l\left(t, x_{t}^{v}, y_{t}^{v}, z_{t}^{v}, v_{t}\right)-l\left(t, x_{t}^{u}, y_{t}^{u}, z_{t}^{u}, u_{t}\right)\right] d t .
\end{aligned}
$$

By applying the Itô formula to $p_{t}\left(x_{t}^{u}-x_{t}^{v}\right)$ and $q_{t}\left(y_{t}^{u}-y_{t}^{v}\right)$, we obtain

$$
\begin{aligned}
& \mathbf{E}\left[p_{T}\left(x_{T}^{v}-x_{T}^{u}\right)\right]=-\mathbf{E} \int_{0}^{T}\left[l_{x}\left(t, x_{t}^{u}, y_{t}^{u}, z_{t}^{u}, u_{t}\right)+b_{x}\left(t, x_{t}^{u}, u_{t}\right) p_{t}\right]\left(x_{t}^{v}-x_{t}^{u}\right) d t \\
& \quad-\mathbf{E} \int_{0}^{T}\left[\sigma_{x}\left(t, x_{t}^{u}, u_{t}\right) P_{t}+f_{x}\left(t, x_{t}^{u}, y_{t}^{u}, z_{t}^{u}, u_{t}\right) q_{t}\right]\left(x_{t}^{v}-x_{t}^{u}\right) d t \\
& \quad+\mathbf{E} \int_{0}^{T}\left(\left[b\left(t, x_{t}^{v}, v_{t}\right)-b\left(t, x_{t}^{u}, u_{t}\right)\right] p_{t}+\left[\sigma\left(t, x_{t}^{v}, v_{t}\right)-\sigma\left(t, x_{t}^{u}, u_{t}\right)\right] P_{t}\right) d t \\
& \mathbf{E}\left[q_{0}\left(y_{0}^{v}-y_{0}^{u}\right)\right]=\mathbf{E}\left[q_{T}\left(y_{T}^{v}-y_{T}^{u}\right)\right] \\
& \quad-\mathbf{E} \int_{0}^{T}\left[l_{y}\left(t, x_{t}^{u}, y_{t}^{u}, z_{t}^{u}, u_{t}\right)+f_{y}\left(t, x_{t}^{u}, y_{t}^{u}, z_{t}^{u}, u_{t}\right) q_{t}\right]\left(y_{t}^{v}-y_{t}^{u}\right) d t \\
& \quad+\mathbf{E} \int_{0}^{T}\left[f\left(t, x_{t}^{v}, y_{t}^{v}, z_{t}^{v}, v_{t}\right)-f\left(t, x_{t}^{u}, y_{t}^{u}, z_{t}^{u}, u_{t}\right)\right] q_{t} d t \\
& \quad-\mathbf{E} \int_{0}^{T}\left[l_{z}\left(t, x_{t}^{u}, y_{t}^{u}, z_{t}^{u}, u_{t}\right)+f_{z}\left(t, x_{t}^{u}, y_{t}^{u}, z_{t}^{u}, u_{t}\right) q_{t}\right]\left(z_{t}^{v}-z_{t}^{u}\right) d t
\end{aligned}
$$

Then

$$
\begin{aligned}
J(v) & -J(u) \geqslant \mathbf{E}\left[q_{T}\left(y_{T}^{v}-y_{T}^{u}\right)\right] \\
& -\mathbf{E} \int_{0}^{T}\left[l_{x}\left(t, x_{t}^{u}, y_{t}^{u}, z_{t}^{u}, u_{t}\right)+b_{x}\left(t, x_{t}^{u}, u_{t}\right) p_{t}\right]\left(x_{t}^{v}-x_{t}^{u}\right) d t \\
& -\mathbf{E} \int_{0}^{T}\left[\sigma_{x}\left(t, x_{t}^{u}, u_{t}\right) P_{t}+f_{x}\left(t, x_{t}^{u}, y_{t}^{u}, z_{t}^{u}, u_{t}\right) q_{t}\right]\left(x_{t}^{v}-x_{t}^{u}\right) d t \\
& +\mathbf{E} \int_{0}^{T}\left(\left[b\left(t, x_{t}^{v}, v_{t}\right)-b\left(t, x_{t}^{u}, u_{t}\right)\right] p_{t}+\left[\sigma\left(t, x_{t}^{v}, v_{t}\right)-\sigma\left(t, x_{t}^{u}, u_{t}\right)\right] P_{t}\right) d t \\
& -\mathbf{E} \int_{0}^{T}\left[l_{y}\left(t, x_{t}^{u}, y_{t}^{u}, z_{t}^{u}, u_{t}\right)+f_{y}\left(t, x_{t}^{u}, y_{t}^{u}, z_{t}^{u}, u_{t}\right) q_{t}\right]\left(y_{t}^{v}-y_{t}^{u}\right) d t \\
& +\mathbf{E} \int_{0}^{T}\left[f\left(t, x_{t}^{v}, y_{t}^{v}, z_{t}^{v}, v_{t}\right)-f\left(t, x_{t}^{u}, y_{t}^{u}, z_{t}^{u}, u_{t}\right)\right] q_{t} d t \\
& -\mathbf{E} \int_{0}^{T}\left[l_{z}\left(t, x_{t}^{u}, y_{t}^{u}, z_{t}^{u}, u_{t}\right)+f_{z}\left(t, x_{t}^{u}, y_{t}^{u}, z_{t}^{u}, u_{t}\right) q_{t}\right]\left(z_{t}^{v}-z_{t}^{u}\right) d t \\
& +\mathbf{E} \int_{0}^{T}\left[l\left(t, x_{t}^{v}, y_{t}^{v}, z_{t}^{v}, v_{t}\right)-l\left(t, x_{t}^{u}, y_{t}^{u}, z_{t}^{u}, u_{t}\right)\right] d t .
\end{aligned}
$$

We remark that $q_{T}\left(y_{T}^{v}-y_{T}^{u}\right)=-k_{T}\left(x_{T}^{v}-x_{T}^{u}\right)$, then by applying the Itô formula to $k_{t}\left(y_{t}^{v}-y_{t}^{u}\right)$, we have

$$
\mathbf{E}\left[k_{T}\left(x_{T}^{v}-x_{T}^{u}\right)\right]=-\mathbf{E} \int_{0}^{T}\left[b_{x}\left(t, x_{t}^{u}, u_{t}\right) k_{t}+\sigma_{x}\left(t, x_{t}^{u}, u_{t}\right) K_{t}\right]\left(x_{t}^{v}-x_{t}^{u}\right) d t
$$




$$
+\mathbf{E} \int_{0}^{T}\left(\left[b\left(t, x_{t}^{v}, v_{t}\right)-b\left(t, x_{t}^{u}, u_{t}\right)\right] k_{t}+\left[\sigma\left(t, x_{t}^{v}, v_{t}\right)-\sigma\left(t, x_{t}^{u}, u_{t}\right)\right] K_{t}\right) d t
$$

Then

$$
\begin{aligned}
J(v) & -J(u) \\
\geqslant & \mathbf{E} \int_{0}^{T}\left[H\left(t, x_{t}^{u}, y_{t}^{u}, z_{t}^{u}, u_{t}, p_{t}, q_{t}, k_{t}\right)-H\left(t, x_{t}^{v}, y_{t}^{v}, z_{t}^{v}, v_{t}, p_{t}, q_{t}, k_{t}\right)\right] d t \\
& +\mathbf{E} \int_{0}^{T} H_{x}\left(t, x_{t}^{u}, y_{t}^{u}, z_{t}^{u}, u_{t}, p_{t}, q_{t}, k_{t}\right)\left(x_{t}^{v}-x_{t}^{u}\right) d t \\
& +\mathbf{E} \int_{0}^{T} H_{y}\left(t, x_{t}^{u}, y_{t}^{u}, z_{t}^{u}, u_{t}, p_{t}, q_{t}, k_{t}\right)\left(y_{t}^{v}-y_{t}^{u}\right) d t \\
& +\mathbf{E} \int_{0}^{T} H_{z}\left(t, x_{t}^{u}, y_{t}^{u}, z_{t}^{u}, u_{t}, p_{t}, q_{t}, k_{t}\right)\left(z_{t}^{v}-z_{t}^{u}\right) d t .
\end{aligned}
$$

Since the Hamiltonian $H$ is concave with respect to $(x, y, z, u)$, we have

$$
\begin{aligned}
& \mathbf{E} \int_{0}^{T}\left[H\left(t, x_{t}^{v}, y_{t}^{v}, z_{t}^{v}, v_{t}, p_{t}, q_{t}, k_{t}\right)-H\left(t, x_{t}^{u}, y_{t}^{u}, z_{t}^{u}, u_{t}, p_{t}, q_{t}, k_{t}\right)\right] d t \\
& \leqslant \mathbf{E} \int_{0}^{T} H_{x}\left(t, x_{t}^{u}, y_{t}^{u}, z_{t}^{u}, u_{t}, p_{t}, q_{t}, k_{t}\right)\left(x_{t}^{v}-x_{t}^{u}\right) d t \\
& \quad+\mathbf{E} \int_{0}^{T} H_{y}\left(t, x_{t}^{u}, y_{t}^{u}, z_{t}^{u}, u_{t}, p_{t}, q_{t}, k_{t}\right)\left(y_{t}^{v}-y_{t}^{u}\right) d t \\
& \quad+\mathbf{E} \int_{0}^{T} H_{z}\left(t, x_{t}^{u}, y_{t}^{u}, z_{t}^{u}, u_{t}, p_{t}, q_{t}, k_{t}\right)\left(z_{t}^{v}-z_{t}^{u}\right) d t \\
& \quad+\mathbf{E} \int_{0}^{T} H_{v}\left(t, x_{t}^{u}, y_{t}^{u}, z_{t}^{u}, u_{t}, p_{t}, q_{t}, k_{t}\right)\left(v_{t}-u_{t}\right) d t
\end{aligned}
$$

or, equivalently,

$$
\begin{aligned}
& \mathbf{E} \int_{0}^{T} H_{v}\left(t, x_{t}^{u}, y_{t}^{u}, z_{t}^{u}, u_{t}, p_{t}, q_{t}, k_{t}\right)\left(u_{t}-v_{t}\right) d t \\
& \leqslant \mathbf{E} \int_{0}^{T}\left[H\left(t, x_{t}^{u}, y_{t}^{u}, z_{t}^{u}, u_{t}, p_{t}, q_{t}, k_{t}\right)-H\left(t, x_{t}^{v}, y_{t}^{v}, z_{t}^{v}, v_{t}, p_{t}, q_{t}, k_{t}\right)\right] d t \\
& \quad+\mathbf{E} \int_{0}^{T} H_{x}\left(t, x_{t}^{u}, y_{t}^{u}, z_{t}^{u}, u_{t}, p_{t}, q_{t}, k_{t}\right)\left(x_{t}^{v}-x_{t}^{u}\right) d t \\
& \quad+\mathbf{E} \int_{0}^{T} H_{y}\left(t, x_{t}^{u}, y_{t}^{u}, z_{t}^{u}, u_{t}, p_{t}, q_{t}, k_{t}\right)\left(y_{t}^{v}-y_{t}^{u}\right) d t \\
& \quad+\mathbf{E} \int_{0}^{T} H_{z}\left(t, x_{t}^{u}, y_{t}^{u}, z_{t}^{u}, u_{t}, p_{t}, q_{t}, k_{t}\right)\left(z_{t}^{v}-z_{t}^{u}\right) d t .
\end{aligned}
$$

Then

$$
J(v)-J(u) \geqslant \mathbf{E} \int_{0}^{T} H_{v}\left(t, x_{t}^{u}, y_{t}^{u}, z_{t}^{u}, u_{t}, p_{t}, q_{t}, k_{t}\right)\left(u_{t}-v_{t}\right) d t .
$$

The necessary conditions of optimality (38) imply $J(v)-J(u) \geqslant 0$. The theorem is proved. 
6. Necessary and sufficient conditions of optimality in the global form. In this section, we give necessary and sufficient conditions of optimality in the global form.

Theorem 3 (Necessary conditions of optimality in the global form). Suppose that $H$ is concave with respect to the control variable $v$. Let $u$ be an optimal control minimizing the functional $J$ over $\mathscr{U}$ and $\left(x_{t}, y_{t}, z_{t}\right)$ the corresponding optimal trajectory. Then there are three unique progressively measurable processes $p, q$, and $k$, which are, respectively, solutions of stochastic differential equations (30), (31), and (32), such that

$$
H\left(t, x_{t}, y_{t}, z_{t}, u_{t}, p_{t}, q_{t}, k_{t}\right)=\max _{v \in U} H\left(t, x_{t}, y_{t}, z_{t}, v, p_{t}, q_{t}, k_{t}\right), \text { a.e., a.s. }
$$

$\mathrm{P}$ r o o f. The condition that $H$ is concave in the control variable $u$ implies that $-H$ is convex with respect to the same argument. Furthermore the map $v \mapsto-H(t, x, y, z, v, p, q, k)$ is continuous, Gâteaux-differentiable, with continuous differential. Then by applying the convex optimization principle (see [14, Proposition 2.1, p. 45]), we have

$u_{t}$ minimizes $-H \Longleftrightarrow-H_{v}\left(t, x_{t}, y_{t}, z_{t}, u_{t}, p_{t}, q_{t}, k_{t}\right)\left(v_{t}-u_{t}\right) \geqslant 0 \quad \forall v \in \mathscr{U}$.

Or, equivalently,

$u_{t}$ maximizes $H \Longleftrightarrow H_{v}\left(t, x_{t}, y_{t}, z_{t}, u_{t}, p_{t}, q_{t}, k_{t}\right)\left(u_{t}-v_{t}\right) \geqslant 0 \quad \forall v \in \mathscr{U}$.

From the above property and Theorem 1, the proof is completed.

Theorem 4 (Sufficient conditions of optimality in global form). Assume that $g(\cdot)$ and $h(\cdot)$ are convex and the map $(x, y, z, v) \mapsto$ $H(t, x, y, z, v, p, q, k)$ is concave. Then $u$ is an optimal control if it satisfies (39).

P r o o f. From (40), it is easy to see that if $u$ satisfies (39), then $u$ satisfies (38). Consequently, from Theorem 2, $u$ is an optimal control.

7. Model with constant terminal condition. Consider a special case of problem (1)-(3) studied in the previous sections, where the terminal condition $y_{T}=\xi$ is an $n$-dimensional $\mathscr{F}_{T}$-measurable random vector such that

$$
\mathbf{E}|\xi|^{2}<\infty
$$

Then the terminal condition in (16) is $\widetilde{y}_{T}=0$, so that $\mathbf{E}\left[q_{T} \widetilde{y}_{T}\right]=0$ and equation (35) becomes

$$
\begin{aligned}
\mathbf{E}\left[q_{0} \widetilde{y}_{0}\right]= & -l_{y}\left(t, x_{t}, y_{t}, z_{t}, u_{t}\right) \widetilde{y}_{t}+f_{x}\left(t, x_{t}, y_{t}, z_{t}, u_{t}\right) q_{t} \widetilde{x}_{t} \\
& +f_{v}\left(t, x_{t}, y_{t}, z_{t}, u_{t}\right) q_{t}\left(v_{t}-u_{t}\right)-l_{z}\left(t, x_{t}, y_{t}, z_{t}, u_{t}\right) \widetilde{z}_{t} .
\end{aligned}
$$


Consequently, from the variational inequality (36), we have

$$
0 \leqslant \widetilde{H}_{v}(t, x, y, z, v, p, q)\left(v_{t}-u_{t}\right),
$$

where the Hamiltonian $\widetilde{H}$, in this case, is defined from $[0, T] \times \mathbf{R}^{n} \times \mathbf{R}^{m} \times$ $\mathscr{M}_{m \times}(\mathbf{R}) \times U \times \mathbf{R}^{n} \times \mathbf{R}^{m}$ into $\mathbf{R}$ by

$$
\widetilde{H}(t, x, y, z, v, p, q)=-l(t, x, y, z, v)-b(t, x, v) p-\sigma(t, x, v) P-f(t, x, y, z, v) q .
$$

In this case, we have only two adjoint processes given by

$$
d p_{t}=\widetilde{H}_{x}\left(t, x_{t}, y_{t}, z_{t}, u_{t}, p_{t}, q_{t}\right) d t+P_{t} d W_{t}, \quad p_{T}=g_{x}\left(x_{T}\right),
$$

and

$$
\begin{aligned}
d q_{t} & =-\widetilde{H}_{y}\left(t, x_{t}, y_{t}, z_{t}, u_{t}, p_{t}, q_{t}\right) d t-\widetilde{H}_{z}\left(t, x_{t}, y_{t}, z_{t}, u_{t}, p_{t}, q_{t}\right) d W_{t} \\
q_{0} & =h_{y}\left(y_{0}\right) .
\end{aligned}
$$

With the Hamiltonian $\widetilde{H}$ and the adjoint processes $p$ and $q$, it is easy to see by using the same proofs, that Theorems $1-4$ are valid, without the processes $k$.

8. Application to cash flow valuation. Modelling and controlling cash-flow processes of a firm or a project like, e.g., pricing and managing an insurance contract is a class of problems, where FBSDEs provide a natural set up and a powerful tool. In this section we shall give an example of such a situation arising in the pricing of a simple insurance contract. This example is taken from [4].

A policyholder at an insurance company has paid premiums that at time 0 have accumulated to the sum $m_{0}$. The money are invested in an asset portfolio with wealth $\left(x_{t}\right)_{t \in[0, T]}$ managed by the insurance company under a time interval $[0, T]$. At each instant $t \in[0, T]$, the policyholder is ought to receive an amount $c_{t} x_{t}$. The present value (price) of the cash stream $\left(c_{s} x_{s}\right)_{t \leqslant s \leqslant T}$, discounted to time $t$ with a discount factor (deflator) $\exp \left\{-\int_{0}^{t} \lambda_{s} d s\right\}$, where $\lambda_{t}$ is assumed nonnegative, bounded, and deterministic, is given by

$$
y_{t}=\mathbf{E}\left[\int_{t}^{T} e^{-\int_{0}^{s} \lambda_{r} d r} c_{s} x_{s} d s \mid \mathscr{F}_{t}\right] .
$$

Assume that the portfolio is invested in a simple Black-Scholes market model consisting of a risk-free asset (for example, a bond or a bank account) with a short interest rate $r_{t}$ assumed bounded and deterministic and a risky asset evolving as a geometric Brownian motion with rate of return $\mu_{t}$ and volatility $\sigma_{t}$, both assumed bounded and deterministic functions of time, with $\sigma_{t} \geqslant \varepsilon>0$ for all $t \in[0, T]$. In this market the wealth process $\left(x_{t}\right)_{t}$ is governed by the dynamics given by

$$
d x_{t}=\left(r_{t} x_{t}+\rho_{t} u_{t}\right) d t+\sigma_{t} u_{t} d W_{t}, \quad x_{0}=m_{0},
$$


where $u_{t}$ is the amount invested in the risky asset and $\rho_{t}=\mu_{t}-r_{t}$ is the risk premium held for this investment.

The insurance company allocates the amounts $\left(u_{t}\right)$ in order to come close to the following target at time $T$ : Find admissible strategies $(c, u)$ which maximize the policyholder's preferences represented by the utility function $F$ of the cash streams, discounted at his personal discount rate $\beta$, assumed constant, and minimize the variance of the terminal wealth, under the condition that the total amount ought to be paid out is equal to the total premium $m_{0}$ :

$$
\left[\begin{array}{l}
\max _{(c, u)} \mathbf{E}\left[\int_{0}^{T} e^{-\beta t} F\left(c_{t} x_{t}\right) d t-\left(x_{T}-\mathbf{E} x_{T}\right)^{2}\right] \\
\text { constrained by } \mathbf{E} x_{T}=d \text { and } \mathbf{E}\left[\int_{0}^{T} e^{-\int_{0}^{t} \lambda_{s} d s} c_{s} x_{s} d s\right]=m_{0} .
\end{array}\right.
$$

Using the Lagrange multiplier method, the problem can be reduced to the following unconstrained control problem:

$$
\max _{(c, u)} \mathbf{E}\left[\int_{0}^{T} e^{-\beta t} F\left(c_{t} x_{t}\right) d t-\frac{\delta}{2}\left(x_{T}-a\right)^{2}+\theta\left(y_{0}-d\right)\right],
$$

where $\delta$ and $\theta$ are the relative weights of hitting the target and

$$
y_{0}=\mathbf{E}\left[\int_{0}^{T} e^{-\int_{0}^{s} \lambda_{r} d r} c_{s} x_{s} d s \mid \mathscr{F}_{0}\right]
$$

is the total value of the stream of cash flows discounted to time zero.

We need the following definition of admissible strategies suitable for our problem.

D e f i n i t i o n 2. An admissible strategy is a pair of $\left(\mathscr{F}_{t}\right)_{t \geqslant 0}$-adapted processes $(c, u)$ such that equation (45) has a strong solution $\left(x_{t}\right)_{t \in[0, T]}$ that satisfies

$$
\mathbf{E} \int_{0}^{T}\left|x_{t}\right|^{2} d t<\infty
$$

and

$$
\mathbf{E}\left(\int_{0}^{T} e^{-\int_{0}^{t} \lambda_{s} d s} c_{t} x_{t} d t\right)^{2}<\infty .
$$

Now, for each admissible strategy $(c, u)$, the $\left(\mathscr{F}_{t}\right)_{t \geqslant 0}$-adapted value process $\left(y_{t}\right)_{t}$ in equation (44) satisfies the following BSDE:

$$
d y_{t}=\left(\lambda_{t} y_{t}-c_{t} x_{t}\right) d t+z_{t} d W_{t}, \quad y_{T}=0,
$$

where $\left(z_{t}\right)_{t}$ is $\left(\mathscr{F}_{t}\right)_{t \geqslant 0}$-adapted and square-integrable with respect to $d t \times \mathbf{P}$ over $[0, T] \times \Omega$. 
Indeed, set

$$
\Lambda_{t}=\exp \left\{-\int_{0}^{t} \lambda_{s} d s\right\}, \quad M_{t}=\mathbf{E}\left[\int_{0}^{T} \Lambda_{s} c_{s} x_{s} d s \mid \mathscr{F}_{t}\right] .
$$

Now, by (48) it follows that $M_{t}$ is a square-integrable martingale adapted to $\left(\mathscr{F}_{t}\right)_{t \geqslant 0}$. Therefore, by the Martingale Representation Theorem, there exists an a.s. unique $\left(\mathscr{F}_{t}\right)_{t \geqslant 0}$-adapted stochastic process $\varphi_{s}$ such that

$$
M_{t}=M_{0}+\int_{0}^{t} \varphi_{s} d W_{s}
$$

where $\varphi_{s}$ is an $\left(\mathscr{F}_{t}\right)_{t \geqslant 0}$-adapted process such that $\mathbf{E} \int_{0}^{T} \varphi_{s}^{2} d s<\infty$. Now, we can write $y_{t}$ as follows:

$$
y_{t}=\frac{M_{t}}{\Lambda_{t}}-\frac{1}{\Lambda_{t}} \int_{0}^{t} \Lambda_{s} c_{s} x_{s} d s
$$

Therefore, $y_{t}$ satisfies

$$
d y_{t}=\left(\lambda_{t} y_{t}-c_{t} x_{t}\right) d t+\frac{\varphi_{t}}{\Lambda_{t}} d W_{t}
$$

Setting $z_{t}=\varphi_{t} / \Lambda_{t}$, we get that $\left(z_{t}\right)_{t}$ is $\left(\mathscr{F}_{t}\right)_{t \geqslant 0}$-adapted and squareintegrable with respect to $d t \times \mathbf{P}$ over $[0, T] \times \Omega$ and that $\left(y_{t}\right)_{t}$ satisfies equation (49).

Hence, equation (45)-(49) satisfied by $(x, y, z)$ is an FBSDE.

Assume that the policyholder's utility function is of HARA (hyperbolic absolute risk aversion) type. That is, $F(X)=X^{\gamma} / \gamma$, where $\gamma \in(0,1)$.

We want to solve the following optimization problem:

$$
\max _{(c, u)} \mathbf{E}\left[g\left(x_{T}\right)+h\left(y_{0}\right)+\int_{0}^{T} e^{-\beta t} \frac{\left(c_{t} x_{t}\right)^{\gamma}}{\gamma} d t\right],
$$

where

$$
g(x)=-\frac{\delta}{2}(x-a)^{2}, \quad h(y)=\theta(y-d),
$$

and $(x, y)$ is the solution of the linear FBSDE (45), (49).

Applying the results of Section 7, the Hamiltonian of our control problem is

$$
H(t, x, y, x, u, c, p, q)=e^{-\beta t} \frac{(c x)^{\gamma}}{\gamma}+\left(r_{t} x+\rho_{t} u\right) p+\sigma_{t} u P+\left(\lambda_{t} y-c x\right) q,
$$

and the system of adjoint equations (42), (43) becomes

$$
\begin{aligned}
d p_{t} & =-\left\{r_{t} p_{t}+e^{-\beta t} c_{t}^{\gamma} x_{t}^{\gamma-1}-c_{t} q_{t}\right\} d t+P_{t} d W_{t}, \\
p_{T} & =g_{x}\left(x_{T}\right)=-\delta\left(x_{T}-a\right) .
\end{aligned}
$$




$$
\begin{aligned}
d q_{t} & =\lambda_{t} q_{t} d t, \\
q_{0} & =h_{y}\left(y_{0}\right)=\theta .
\end{aligned}
$$

Now, let $(\widehat{c}, \widehat{u})$ be a candidate for an optimal strategy, and let $\left(\widehat{x}_{t}, \widehat{y}_{t}, \widehat{z}_{t}\right)$ be the corresponding solution of our FBSDE (45), (49) with associated solution $\left(\widehat{p}_{t}, \widehat{q}_{t}\right)$. The value of $c$ which maximizes $H\left(t, \widehat{x}_{t}, \widehat{y}_{t}, \widehat{z}_{t}, \cdot, \widehat{u}, \widehat{p}_{t}, \widehat{q}_{t}\right)$ satisfies

$$
\widehat{c}_{t}=\left(e^{-\beta t} \widehat{x}_{t}^{1-\gamma} \widehat{q}_{t}\right)^{1 /(\gamma-1)} .
$$

Since $\left(\lambda_{t}\right)_{t \geqslant 0}$ is assumed nonnegative, condition (48) is satisfied. Furthermore, since the term involving $u$ in the Hamiltonian is linear, the $u$ coefficient should vanish, i.e.,

$$
\widehat{P}_{t}=-\frac{\rho_{t}}{\sigma_{t}} \widehat{p}_{t}
$$

Therefore, by (54) and (55), $\left(\widehat{p}_{t}, \widehat{q}_{t}\right)$ satisfies the following decoupled FBSDE:

$$
\begin{aligned}
& d p_{t}=-r_{t} p_{t} d t-\frac{\rho_{t}}{\sigma_{t}} p_{t} d W_{t}, \quad p_{T}=g_{x}\left(x_{T}\right), \\
& d q_{t}=\lambda_{t} q_{t} d t, \quad q_{0}=h_{y}\left(y_{0}\right) .
\end{aligned}
$$

Equation (57) yields a unique solution

$$
\widehat{q}_{t}=h_{y}\left(\widehat{y}_{0}\right) \exp \left\{\int_{0}^{t} \lambda_{s} d s\right\}
$$

Let us look for a solution to equation (56) of the form

$$
p_{t}=f(t) \widehat{x}_{t}+g(t),
$$

for smooth deterministic functions $f$ and $g$.

This choice is motivated by the fact that the terminal value $\widehat{p}_{T}$ is linear in $\widehat{x}_{T}$.

Using the Itô lemma and identifying the coefficients in equations (45) and (56), the functions $f$ and $g$ must satisfy

$$
\begin{gathered}
\left(\dot{f}(t)+2 r_{t} f(t)\right) \widehat{x}_{t}+\rho_{t} \widehat{u}_{t} f(t)+\dot{g}(t)+r_{t} g(t)=0, \\
-\frac{\rho_{t}}{\sigma_{t}}\left(f(t) \widehat{x}_{t}+g(t)\right)=f(t) \sigma_{t} \widehat{u}_{t} .
\end{gathered}
$$

Hence,

$$
\begin{aligned}
& \dot{f}(t)=\left(\frac{\rho_{t}^{2}}{\sigma_{t}^{2}}-2 r_{t}\right) f(t), \quad f(T)=-\delta, \\
& \dot{g}(t)=\left(\frac{\rho_{t}^{2}}{\sigma_{t}^{2}}-r_{t}\right) g(t), \quad g(T)=\delta a .
\end{aligned}
$$


The solutions to these equations are

$$
\begin{array}{ll}
f(t)=-\delta \exp \left\{\int_{t}^{T}\left(\frac{\rho_{s}^{2}}{\sigma_{s}^{2}}-2 r_{s}\right) d s\right\}, & t \in[0, T], \\
g(t)=\delta a \exp \left\{\int_{t}^{T}\left(\frac{\rho_{s}^{2}}{\sigma_{s}^{2}}-r_{s}\right) d s\right\}, & t \in[0, T] .
\end{array}
$$

Finally,

$$
\widehat{u}_{t}=-\frac{\rho_{t}}{\sigma_{t}^{2}} \widehat{x}_{t}-\frac{g(t)}{f(t) \sigma_{t}^{2}} .
$$

Since $\widehat{u}_{t}$ is linear in $\widehat{x}_{t}$, it leads to a linear SDE with bounded coefficients for $\widehat{x}$, and therefore it satisfies (47).

Summing up, an optimal admissible strategy $(\widehat{c}, \widehat{u})$ for problem (50) subject to the dynamics (45) and (49) is given by (54) and (64).

Acknowledgments. The author thanks the referee who offered many useful remarks and suggestions that improved the manuscript.

\section{REFERENCES}

1. Antonelli F. Backward-forward stochastic differential equations. - Ann. Appl. Probab., 1993, v. 3, № 3, p. 777-793.

2. Antonelli F., Ma. J. Weak solutions of forward-backward SDE's. - Stoch. Anal. Appl., 2003, v. 21, № 3, p. 493-514.

3. Аркин В.И., Саксонов М.Т. Необходимые условия оптимальности в задачах управления стохастическими дифференциальными уравнениями. - Докл. АН CCCP, 1979, т. 244, № 1, c. 11-15.

4. Armerin F. Aspects of cash flow valuation. Ph.D. Thesis. Stockholm: KTH, 2004.

5. Bahlali S., Mezerdi B. A general stochastic maximum principle for singular control problems. - Electron. J. Probab., 2005, v. 10, paper № 30, p. 988-1004.

6. Bahlali S., Mezerdi B., Djehiche, B. Approximation and optimality necessary conditions in relaxed stochastic control problems. - J. Appl. Math. Stoch. Anal., 2006, Art. ID 72762 , p. 1-23.

7. Bahlali S., Labed B. Necessary and sufficient conditions of optimality for optimal control problem with initial and terminal costs. - Random Oper. Stoch. Equ., 2006, v. 14, № 3, p. 291-301.

8. Bahlali S., Djehiche B., Mezerdi B. The relaxed stochastic maximum principle in singular control of diffusions. - SIAM J. Control Optim., 2007, v. 46, № 2, p. 427444.

9. Bensoussan A. Lectures on stochastic control. - Lecture Notes in Math., 1982, v. 972, p. $1-62$.

10. Cadenillas A., Karatzas I. The stochastic maximum principle for linear convex optimal control with random coefficients. - SIAM J. Control Optim., 1995, v. 33, № 2, p. 590624.

11. Delarue F. On the existence and uniqueness of solutions to FBSDEs in a nondegenerate case. - Stochastic Process. Appl., 2002, v. 99, № 2, p. 209-286.

12. Dokuchaev N., Zhou X. Y. Stochastic controls with terminal contingent conditions. J. Math. Anal. Appl., 1999, v. 238, № 1, p. 143-165.

13. Douglas J., Ma J., Protter P. Numerical methods for forward-backward stochastic differential equations. - Ann. Appl. Probab., 1996, v. 6, № 3, p. 940-968. 
14. Экланд И., Темам Р. Выпуклый анализ и вариационные проблемы. М.: Мир, 1979, 399 c.

15. Backward Stochastic Differential Equations. Ed. by N. El Karoui and L. Mazliak. Harlow: Longman, 1997, $221 \mathrm{p}$.

16. El Karoui N., Peng S., Quenez M. C. Backward stochastic differential equations in finance. - Math. Finance, 1997, v. 7, № 1, p. 1-71.

17. Fuhrman M., Tessitore G. Existence of optimal stochastic controls and global solutions of forward-backward stochastic differential equations. - SIAM J. Control Optim., 2004, v. 43, № 3, p. 813-830.

18. Haussmann U.G. General necessary conditions for optimal control of stochastic systems. - Stochastic Systems: Modeling, Identification and Optimization (Lexington, 1975). Amsterdam: North-Holland, 1976, p. 30-48. (Math. Programming Stud., v. 6.)

19. Haussmann U. G. A Stochastic Maximum Principle for Optimal Control of Diffusions. Harlow/New York: Longman/Wiley, 1986, 109 p.

20. $\mathrm{Hu} Y$. On the solution of forward-backward SDEs with monotone and continuous coefficients. - Nonlinear Anal., 2000, v. 42, p. 1-12.

21. Hu Y., Peng S. Solution of forward-backward stochastic differential equations. Probab. Theory Related Fields, 1995, v. 103, № 2, p. 273-283.

22. Hu Y., Yong J. Forward-backward stochastic differential equations with nonsmooth coefficients. - Stochastic Process. Appl., 2000, v. 87, № 1, p. 93-106.

23. Kushner H. J. Necessary conditions for continuous parameter stochastic optimization problems. - SIAM J. Control Optim., 1972, v. 10, p. 550-565.

24. Ma J., Protter P., Yong J. Solving forward-backward stochastic differential equations explicitly - a four step scheme. - Probab. Theory Related Fields, 1994, v. 98, № 3, p. 339-359.

25. Ma J., Yong J. Forward-backward stochastic differential equations and their applications. - Lecture Notes in Math., 1999, v. 1702, p. 1-270.

26. Ma J., Zhang J. Representation theorems for backward stochastic differential equations. - Ann. Appl. Probab., 2002, v. 12, № 4, p. 1390-1418.

27. Pardoux É., Peng S. Adapted solutions of a backward stochastic differential equations. - Systems Control Lett., 1990, v. 14, № 1, p. 55-61.

28. Pardoux É., Tang S. Forward-backward stochastic differential equations and quasilinear parabolic PDEs. - Probab. Theory Related Fields, 1999, v. 114, № 2, p. 123-150.

29. Peng S. A general stochastic maximum principle for optimal control problems. SIAM J. Control Optim., 1990, v. 28, № 4, p. 966-979.

30. Peng S. Backward stochastic differential equations and application to optimal control. - Appl. Math. Optim., 1993, v. 27, № 2, p. 125-144.

31. Peng $S$., $W u Z$. Fully coupled forward-backward stochastic differential equations and applications to optimal control. — SIAM J. Control Optim., 1999, v. 37, № 3, p. 825843.

32. $W u Z$. Maximum principle for optimal control problem of fully coupled forwardbackward stochastic systems. - Systems Sci. Math. Sci., 1998, v. 11, № 3, p. 249-259.

33. $X u W$. Stochastic maximum principle for optimal control problem of forward and backward system. - J. Austral. Math. Soc. Ser. B, 1995, v. 37, № 2, p. 172-185.

34. Yong J. Finding adapted solutions of forward-backward stochastic differential equations: method of continuation. - Probab. Theory Related Fields, 1997, v. 107, № 4, p. $537-572$.

Поступила в редакцию 25.IV.2006

Исправленный вариант 27.IV.2008 